WM-02-101

November 5, 2018

\title{
Large Electric Dipole Moments of Heavy Neutrinos
}

\author{
Marc Sher 円 \\ Nuclear and Particle Theory Group \\ Physics Department \\ College of William and Mary, Williamsburg, VA 23187, USA \\ Shuquan Nie f \\ Physics Department, Concordia University, 1455 De Maisonneuve Blvd. W. \\ Montreal, Quebec, Canada, H3G 1 M8
}

\begin{abstract}
In many models of CP violation, the electric dipole moment (EDM) of a heavy charged or neutral lepton could be very large. We present an explicit model in which a heavy neutrino EDM can be as large as $10^{-16} \mathrm{e}-\mathrm{cm}$, or even a factor of ten larger if fine-tuning is allowed, and use an effective field theory argument to show that this result is fairly robust. We then look at the production cross section for these neutrinos, and by rederiving the Bethe-Block formula, show that they could leave an ionization track. It is then noted that the first signature of heavy neutrinos with a large EDM would come from $e^{+} e^{-} \rightarrow \bar{N} N \gamma$, leading to a very large rate for single photon plus missing energy events, and the rate and angular distribution are found. Finally, we look at some astrophysical consequences, including whether these neutrinos could constitute the UHE cosmic rays and whether their decays in the early universe could generate a net lepton asymmetry.
\end{abstract}

\footnotetext{
${ }^{1}$ Email: sher@physics.wm.edu

${ }^{2}$ Email: sxnie@alcor.concordia.ca
} 


\section{Introduction}

Most of the unknown parameters of the Standard Model come from fermion masses and mixing angles. They are put in by hand, and we have no real understanding of their origins. Many models that try to explain their values exist, most involving additional symmetries, but more experimental data will be needed to distinguish between them. Much of the excitement concerning neutrino masses and mixing angles is caused by the hope that their values will facilitate our understanding of the flavor problem.

A potentially valuable source of information about the flavor problem may come from electric and magnetic dipole moments. Just as the Yukawa couplings form a matrix in generation space, the interaction of two fermions with a photon will also be a matrix in generation space. The real and imaginary parts of the diagonal elements will lead to the magnetic and electric dipole moments; the off-diagonal elements wil lead to flavorchanging radiative decays. If one can measure these moments, one would obtain valuable hints of physics beyond the Standard Model and would learn more about the origin of flavor.

This paper is concerned with the electric dipole moments (EDMs) of leptons. There is a possibility of substantial improvement in the experimental bounds on EDMs in the near future. Proposals exist[1] to lower the current bound[2] on the muon's EDM by six orders of magnitude, and substantial improvement in the bound on the electron EDM[3] is possible. Combining limits on the weak dipole moment of the tau with $U(1)$ symmetry $\llbracket$ improves the current bound[5] by a factor of thirty.

How big might one expect the EDMs to be? In the Standard Model, they are negligibly small[6]. However, they can be much larger in extensions of the Standard Model. In multi-Higgs models, the EDM of the muon can easily be as large as $10^{-24} \mathrm{e}-\mathrm{cm}$, within

reach of planned experiments[7]. In leptoquark models, the muon and tau EDM's are 
typically $10^{-24}$ e-cm and $10^{-19}$ e-cm, respectively[8]. Left-right models [9] have a muon EDM which is typically of the order of $10^{-24} \mathrm{e}-\mathrm{cm}$, and in the minimal supersymmetric standard model(MSSM) 10, the electron EDM is above the experimental bounds if the phases are all unity. The point is that a wide variety of models give EDMs that can be observed in the near future.

Babu, Barr and Dorsner 11] have discussed how the EDMs of leptons scale with the lepton masses. In many models, such as the Standard Model and the MSSM, they scale linearly with the mass. However, in a large number of models, such as multi-Higgs, leptoquark and some flavor symmetry models, the EDM scales as the cube of the lepton mass. In these models, the tau EDM will be 5000 times larger than that of the muon (it should be noted that the electron EDM in some of these models receives a two-loop contribution which varies only linearly, and thus it need not be negligible).

Given cubic scaling, the EDM of a heavy lepton (charged or neutral) could be quite large. For example, a $100 \mathrm{GeV}$ heavy lepton would have an EDM a billion times larger than the muon's. If the latter is in the expected range of $10^{-24} \mathrm{e}-\mathrm{cm}$, then such a heavy lepton would have an EDM as large as 0.01 e-fermi. This EDM would then dominate the electromagnetic interactions of these leptons, drastically changing their phenomenology. In particular, if a heavy neutrino acquired such an EDM, it could even leave an observable ionization track.

Although originally motivated by cubic scaling models, the possibility of a heavy lepton having a huge EDM is worth studying in a model-independent way. We will see below that plausible models exist in which a heavy neutrino has a very large EDM, even though these models do not have cubic scaling. In this Article, we will concentrate on the possibility that a heavy neutrino could have a large EDM. In an earlier paper by one of us [12], the differential production cross section for heavy leptons (charged and neutral) with large EDMs was studied, and the fact that heavy neutrinos with large EDMs could 
leave an ionization track was discussed (although no explicit models were mentioned). This Article extends this earlier work substantially. In Section II, we explicitly present a model in which the EDM of a heavy neutrino can be of $O\left(10^{-16}\right)$ e-cm. This will serve as an existence proof that relatively simple models can exist in which this occurs. Current bounds on such large EDMs will be noted. In Section III, the cross section for heavy neutrino production is presented, and the Bethe-Block formula for a large-EDM heavy neutrino traversing matter will be calculated. This extends slightly previous work. The result will be that an ionization track is observable, but one would need to either modify existing detectors or construct a new detector. Since it is unlikely that this would occur without some preliminary evidence that such a large-EDM neutrino exists, we look, in

Section IV, at the process of initial and final state radiation, $e^{+} e^{-} \rightarrow N \bar{N} \gamma$, which would give an enormous rate for single-photon plus missing energy events, and would provide impetus for looking explicitly for large-EDM heavy neutrinos. Finally, in Section V, we discuss whether these heavy neutrinos could be candidates for the ultra-high energy cosmic rays, and also consider the implications for baryogenesis through leptogenesis, and in Section VI, we present our conclusions.

\section{Plausible Models}

Are there plausible models in which a huge EDM for a heavy neutrino occurs? If one does not rely on any particular model, then a simple effective field theory argument can be used. Suppose one assumes that $C P$ violation is due to some sort of new physics at the $\mathrm{TeV}$ scale. Then one can write the effective low-energy, dimension-five Lagrangian as

$$
\mathcal{L}=\frac{c}{\Lambda} \bar{L}_{L} \sigma_{\mu \nu} i \gamma_{5} L_{R} F_{\mu \nu}
$$


If $\Lambda$ is $O(1) \mathrm{TeV}$, and the unknown coefficient is of $O(1)$, then this yields a very large EDM of approximately $10^{-15} \mathrm{e}-\mathrm{cm}$ for the lepton.

Of course, in realistic models, one expects the EDM to occur via a loop, suppressing the unknown coefficient, and there may not even be a dimension-five operator. What about specific models currently in the literature? A model with cubic scaling is that of Bernreuther, Schroder and Pham[13], in which CP violation occurs in the Higgs sector. In this model, the EDM of a heavy lepton (they consider the top quark, but the results are unchanged) is constrained by the electron EDM, and the maximum value is $O\left(10^{-17}\right)$ e-cm. In other models, such as the model of Babu, Barr and Dorsner[11] in which their parameter $c=0$, the electron EDM does not give any such constraints and a large EDM is allowed.

Here, we will provide an explicit example of a model with a large EDM. Consider a model containing singlet fermions, $E_{L, R}$ and $N_{L, R}$ and two charged singlet scalar fields, $h_{i}$. The fermions can couple to the charged scalars, $h_{i}, i=1,2$, in the following form

$$
Y_{i} \bar{E}_{R} N_{L} h_{i}+Z_{i} \bar{E}_{L} N_{R} h_{i}+\text { h.c. }
$$

where $Y_{i}$ and $Z_{i}$ are complex Yukawa couplings and can generally include flavor indices. The $h_{i}$ can also mix with each other and can be diagonalized by a complex unitary matrix $\mathrm{U}: h_{i}=U_{i j} H_{j}$, with mass eigenstates $H_{i}$. Two such charged singlets are necessary so that a phase redefinition cannot eliminate the $\mathrm{CP}$ violation. For simplicity in presentation, we will look at the single $H$ case, and just assume that the phase redefinition does not occur. In this case, the coupling is

$$
\bar{E}\left(S-P \gamma_{5}\right) N H+\text { h.c. }
$$

where $\mathrm{S}$ and $\mathrm{P}$ are arbitrary complex couplings.

It is straightforward to calculate the EDM due to the above interaction. The one-loop 

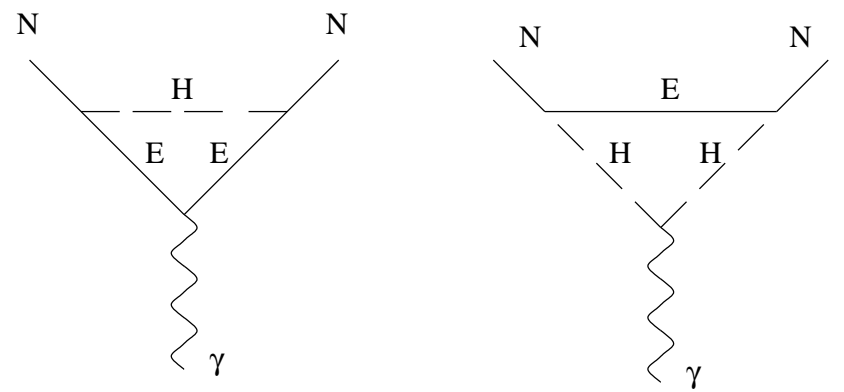

Figure 1: One-loop feynman diagrams contributing to a large EDM for a heavy neutrino.

feynman diagrams are listed in Fig. 1. The EDM is defined at $q^{2}=0$, and is given by

$$
d_{N}\left(q^{2}=0\right)=e \frac{m_{E}}{8 \pi^{2}} \operatorname{Im}\left(S P^{*}\right) \int_{0}^{1} d z \frac{(1-z)(1+2 z)}{-z(1-z) m_{N}^{2}+(1-z) m_{E}^{2}+z m_{H}^{2}}
$$

which, numerically, in units of e-cm, is

$$
3.2 \times 10^{-17}\left(\frac{100 \mathrm{GeV}}{M_{E}}\right) \frac{\operatorname{Im}\left(S P^{*}\right)}{4 \pi} \int_{0}^{1} d z \frac{(1-z)(1+2 z)}{-z(1-z) a^{2}+(1-z)+z b^{2}}
$$

where $a \equiv M_{N} / M_{E}$ and $b \equiv M_{H} / M_{E}$.

The integral is plotted in Fig. 2. We see that EDM's of $10^{-16} \mathrm{e}-\mathrm{cm}$ are quite possible, especially in the region of paramter-space in which the $E$ is heavier than the $N$ or $H$, if one chooses $\frac{\operatorname{Im}\left(S P^{*}\right)}{4 \pi} \sim 1$, and can even be an order of magnitude higher if one allows for some fine-tuning. We have not considered the unlikely region of parameter space in which $M_{N}>M_{E}+M_{H}$, since the width of the $N$ in this case is comparable to its mass, and the definition of the EDM is no longer straightforward.

We thus have presented a model in which the EDM of a heavy neutrino is very large, of $O\left(10^{-16}\right)$ e-cm. While this model is, admittedly, designed to have such a large EDM, its ingredients are very simple, and one can imagine that it may be part of a more complicated model. The main point is that having a heavy neutrino with a large EDM is certainly not excluded, and in this paper we are exploring the phenomenological implications. 


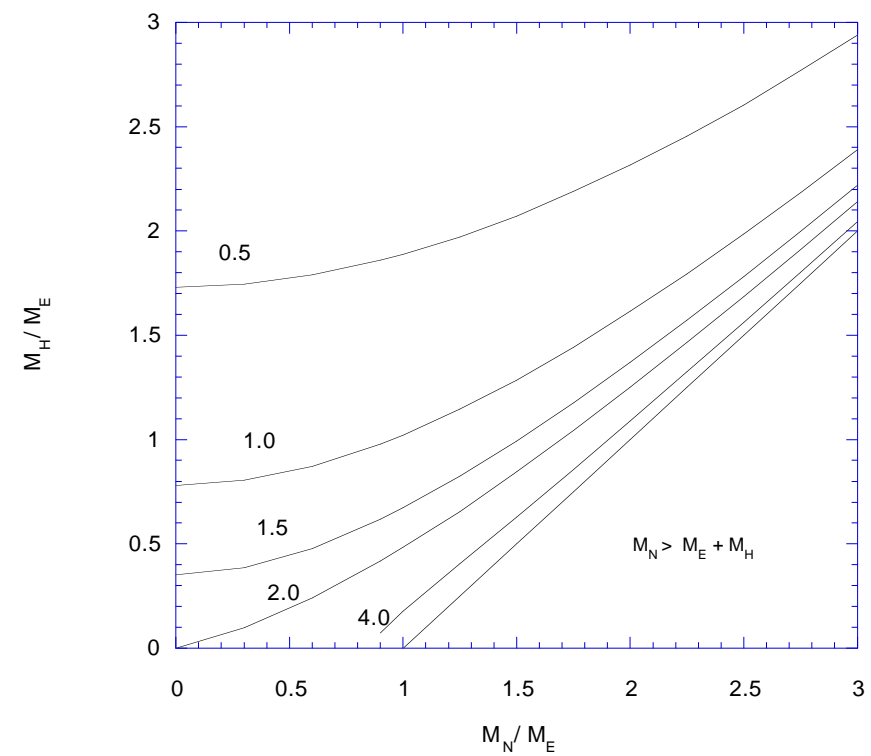

Figure 2: Value of the integral in the expression for the EDM.

Are there any current phenomenological bounds? If the heavy neutrino and charged lepton are part of a fourth chiral fermion family (albeit with a right-handed neutrino), then the $\mathrm{S}$ and $\mathrm{T}$ parameters will cause severe constraints, but if they are part of a vectorlike multiplet, there will be no such constraints. Very explicit models may be constrained, but there are no general bounds. One possible concern would be g-2 of the muon. Here, there will be an effect of a large EDM on the photon propagator. At the lowest order, the feynman diagram is illustrated in Fig. 3. As in the calculation of hadronic contributions to $(g-2)_{\mu}$, we calcualte the effect of a large EDM on $(g-2)_{\mu}$ by the dispersion integral 14

$$
a_{\mu}^{E D M}=\frac{1}{4 \pi^{3}} \int_{4 m_{N}^{2}} d s K(s) \sigma(s)_{e^{+} e^{-} \rightarrow N N},
$$

where $\mathrm{K}(\mathrm{s})$ is given in Ref. [14] and $\sigma(s)_{e^{+} e^{-} \rightarrow N N}$ is given in Ref. [12], as well as below. We cutoff the upper bound of the integral. Letting $m_{N}=100 \mathrm{GeV}, a_{\mu}^{E D M}=$ $5.9 \times 10^{-11}\left(1.3 \times 10^{-10}\right)$ for a cutoff of 1 (10) TeV. This is much smaller than the 


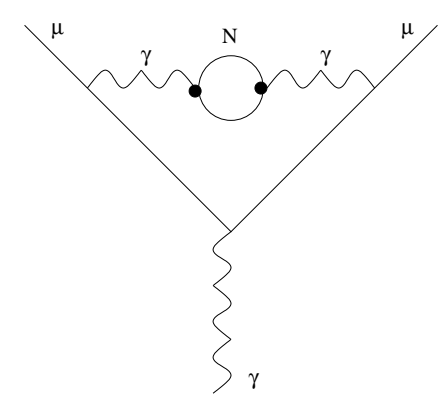

Figure 3: Large EDM contribute to $a_{\mu}$

theoretical and experimental uncertainties, and is thus negligible.

\section{Direct detection}

The most dramatic effect of a large EDM of a heavy neutrino will be in the production cross-section and angular distribution. Escribano and Masso 41 noted that the relevant $U(1)$ invariant effective operator is given by $\bar{L}_{L} \sigma^{\mu \nu} i \gamma_{5} L_{R} B_{\mu \nu}$, where $B_{\mu \nu}$ is the $U(1)$ field tensor. This gives a coupling to the photon, which we define to be the EDM, as well as a coupling to the $Z$ which is the EDM times $\tan \theta_{W}$. We will include this coupling to the $Z$. It turns out that the contribution from the $Z$ has very little effect on the numerical results. In general, one should also include an operator coupling to the $S U(2)$ field tensor, leading to a very different value for the CP-violating coupling to the $Z$. Rather than deal with two parameters, however, we just assume that the latter operator is smaller. If this assumption is false, then it will just make the cross-section even bigger, unless there is fine-tuning. Also, unless the CP-violating coupling to the $Z$ is surprisingly large, it will have very little effect on the results.

A discussion of the differential cross section for a heavy charged lepton can be found in Ref. [12]. Here we are interested in heavy neutrino production. The differential 


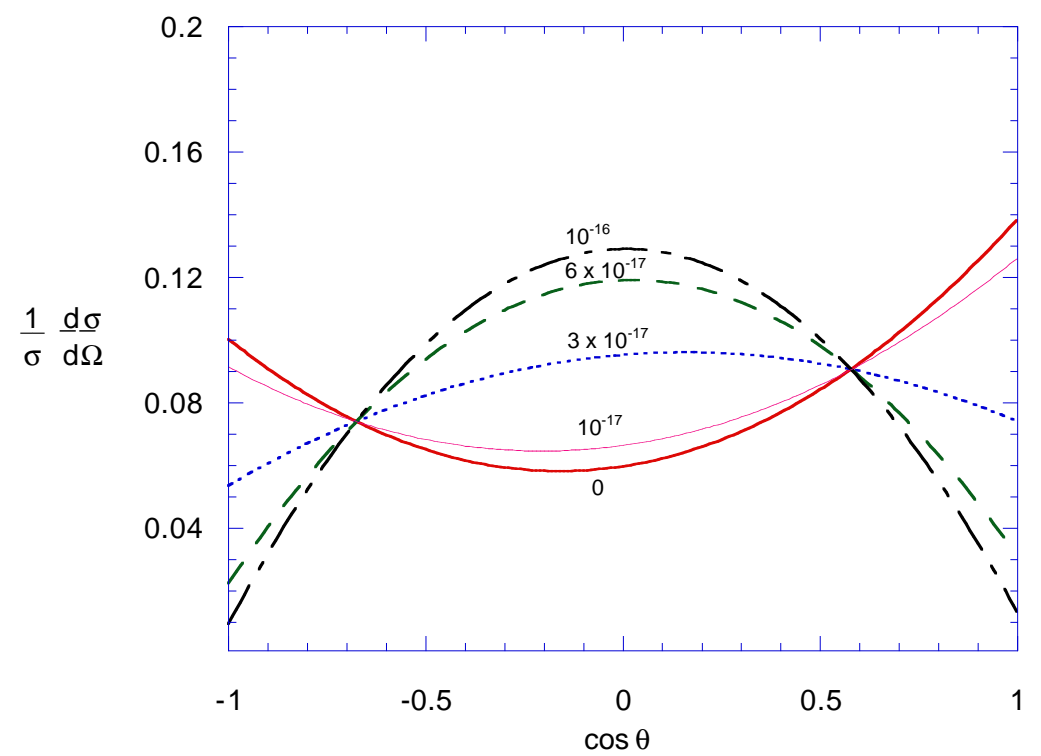

Figure 4: Differential cross section for heavy neutrino production for various EDMs, in units of e-cm, for a lepton mass of $100 \mathrm{GeV}$.

cross-section is given by

$$
\frac{d \sigma}{d \Omega}=\frac{\alpha^{2}}{4 s} \sqrt{1-\frac{4 M^{2}}{s}}\left(A_{1}+\frac{1}{8 \sin ^{4} 2 \theta_{W}} P_{Z Z} A_{2}+\frac{\left(1-4 \sin ^{2} \theta_{W}\right) \tan \theta_{W}}{\sin ^{2} 2 \theta_{W}} P_{\gamma Z} A_{3}\right)
$$

where

$$
\begin{aligned}
& A_{1}=D^{2} s \sin ^{2} \theta\left(1+\frac{4 M^{2}}{s}\right) \\
& A_{2}=1+\cos ^{2} \theta-\frac{4 M^{2}}{s} \sin ^{2} \theta+8 C_{V} \cos \theta+D^{2} s \tan ^{2} \theta_{W}\left(\sin ^{2} \theta+\frac{4 M^{2}}{s}\left(1+\cos ^{2} \theta\right)\right) \\
& A_{3}=4 D^{2} s\left(\sin ^{2} \theta+\frac{4 M^{2}}{s}\left(1+\cos ^{2} \theta\right)\right)
\end{aligned}
$$

where we have dropped the numerically negligible $C_{V}^{2}$ terms, for simplicity.

The differential and total cross-sections are given in Figures 4 and 5, for a heavy neutrino mass of $100 \mathrm{GeV}$. We see that for $D=0$, the usual $1+\cos ^{2} \theta+C \cos \theta$ distribution for a lepton (where the $\cos \theta$ term is due to $\gamma-Z$ interference) is found. For a very large EDM, the distribution is completely dominated by the $\sin ^{2} \theta$ contribution from the 


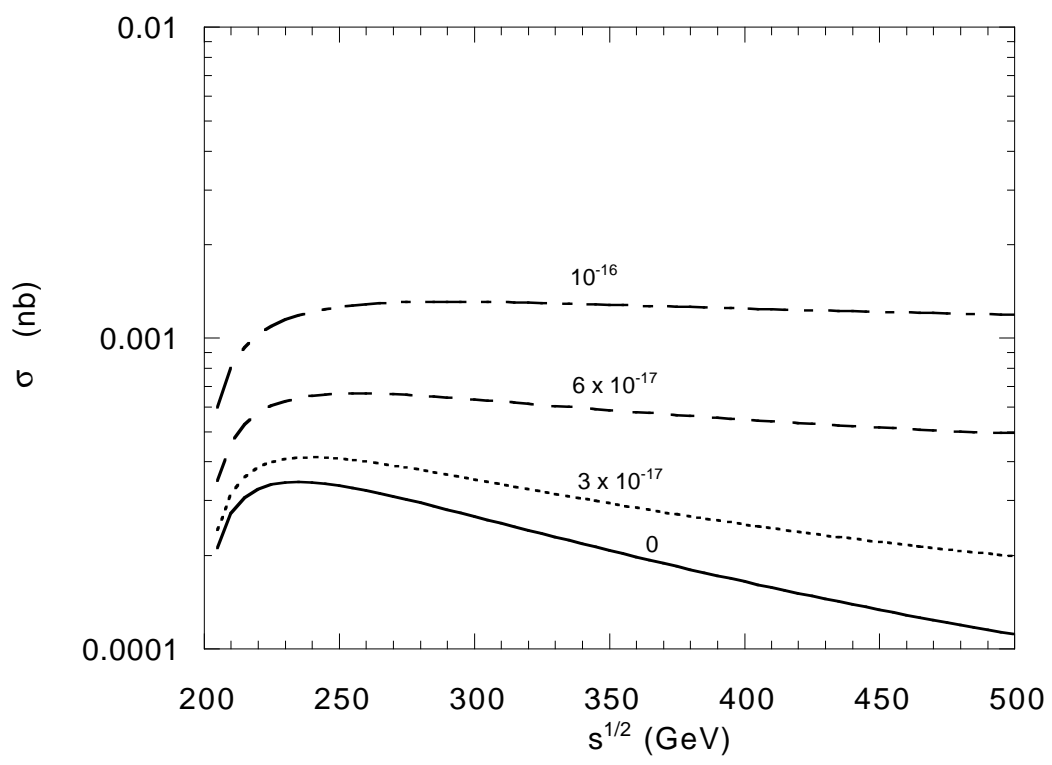

Figure 5: The total cross section for heavy neutrino production for various EDMs, in units of e-cm, for a lepton mass of $100 \mathrm{GeV}$.

electric dipole term. The angular distribution begins to deviate significantly from the $D=0$ distribution for $D$ greater than $3 \times 10^{-17}$, and by $D=3 \times 10^{-16}$ the distribution is very close to $\sin ^{2} \theta$. One can see that EDM's of the size noted earlier will dramatically alter the angular distribution 12 .

Note that since the cross-section varies as $D^{2}$, for an EDM as ridiculously large as 1.0 e-fermi, it would be almost a microbarn!! Cross sections this large will violate unitarity. The unitarity limit can be estimated by setting the relevant effective interaction strength, $\alpha D \sqrt{s}$, equal to unity. For $\sqrt{s}=500 \mathrm{GeV}, D=10^{-15} \mathrm{e}-\mathrm{cm}$, and $\alpha \simeq 1 / 125$, this effective interaction strength is 0.25 . Thus the unitarity limit will not be reached unless the EDM is larger than $10^{-15} \mathrm{e}-\mathrm{cm}$, and so the behavior discussed above will appear for a wide range of parameter space without violating the unitarity bound. Another way of saying this is to note that the larger the EDM, the smaller the scale at which the physics responsible for the effective interaction sets in, and for an EDM larger than $10^{-15} \mathrm{e}-\mathrm{cm}$, that scale is 
less than $\sqrt{s}$.

Production of a fermion with a large EDM has been considered elsewhere. It is given in the context of a top quark EDM by Bernreuther et al. [13], and given in the context of tau-pair production in Ref. [15. This latter paper noted how one can use CP-odd angular correlations to search for a tau EDM, and this method has been used by experimentalists. However, there has not been any discussion of the possibility of a large EDM for heavy neutrinos, and here we see a unique and unusual signature.

What is the purpose of this calculation? After all, it would not seem remotely possible to detect heavy neutrinos directly. This calculation would only be meaningful if the heavy neutrino were heavier than the charged lepton, and could thus be detected via its decay. Here, one could again look for CP-odd correlations, as discussed in Ref. [15]. However, most models have the charged lepton heavier than the neutrino, and thus the decay can only occur through mixing with the very light neutrinos. As discussed in detail in Ref. [16], this mixing could be very small, and these heavy neutrinos could be effectively stable. As we will see below, however, it may be possible to detect these neutrinos initially through final state radiation, and then directly.

Because the heavy neutrino has such a large electric dipole moment, it might be possible to detect it directly through its ionization loss. To estimate whether or not this might be possible, we follow the derivation of the classical Bohr formula outlined in Jackson[17], replacing the electric field from a charge with the electric field from a dipole. This is a classical calculation; if one is not too far above threshold, the neutrino will be moving non-relativistically. Consider a heavy neutrino moving in the $x$-direction, and an electron at an impact parameter $y=b$. The impulse given to the electron is $\Delta \vec{p}=\int_{-\infty}^{\infty} e \vec{E} d t$. The result will depend on the orientation of the dipole. Suppose that the dipole is in the $z$-direction, transverse to the plane of the particle motion and the electron. Then the only nonzero electric field component is in the $z$-direction, and 
$E_{z}=\frac{e D}{4 \pi \epsilon_{o}}\left(b^{2}+v^{2} t^{2}\right)^{-3 / 2}$, where the time of closest approach is defined to be $t=0, v$ is the velocity, and $e D$ is the size of the electric dipole moment. Integrating, one finds that the impulse is $\frac{e^{2} D}{4 \pi \epsilon_{o}} \frac{2}{v b^{2}}$. Suppose that the dipole is in the $y$-direction. Then the electric field components are $E_{x}=\frac{e D}{4 \pi \epsilon_{o} r^{3}}(3 \sin \theta \cos \theta)$ and $E_{y}=\frac{e D}{4 \pi \epsilon_{o} r^{3}}\left(3 \cos ^{2} \theta-2\right)$, where $r^{2}=b^{2}+v^{2} t^{2}$ and $\tan \theta=b / v t$. Integrating, the impulse in the $x$-direction vanishes, as expected by symmetry, and the impulse in the $y$-direction is also $\frac{e^{2} D}{4 \pi \epsilon_{o}} \frac{2}{v b^{2}}$. Finally, if the dipole is in the $x$-direction, the electric field components are $E_{y}=\frac{e D}{4 \pi \epsilon_{o} r^{3}}(3 \sin \theta \cos \theta)$ and $E_{x}=$ $\frac{e D}{4 \pi \epsilon_{o} r^{3}}\left(3 \cos ^{2} \theta-1\right)$. Both of these integrate to zero, so there is no net momentum transfer in this case. Since the orientation is generally arbitrary, and we are only interested in a rough order-of-magnitude estimate, we will take the impulse to be $|\Delta \vec{p}|=\frac{e^{2} D}{4 \pi \epsilon_{o}} \frac{2}{v b^{2}}$. Note that the impulse from an electric charge is just $\frac{2 e^{2}}{4 \pi \epsilon_{o} v b}$, so this result is expected dimensionally. This impulse is then converted into an energy transfer, $\Delta E=|\Delta \vec{p}|^{2} / 2 m$. Jackson notes that the maximum energy transfer is $\Delta E_{\max }=2 m \gamma^{2} v^{2}$, and thus the minimum impact parameter is $b_{\min }^{2}=e^{2} D /\left(m \gamma v^{2}\right)$. The energy loss is obtained by cylindrically integrating over the impact parameter

$$
\frac{d E}{d x}=2 \pi N Z \int_{b_{\text {min }}}^{\infty} \Delta E(b) b d b
$$

and we obtain

$$
\frac{d E}{d x}=2 \pi N_{A}\left(\frac{e^{2}}{4 \pi \epsilon_{o}}\right) D \gamma \frac{Z}{A}
$$

which is the corresponding formula to the Bohr result. Since we are doing a classical calculation, one can set $\gamma=1$. Note that the logarithm in the usual Bethe-Block formula is absent and the electron mass and particle velocity drop out. This is due to the extra power of $b$ in the expression for the impulse, which arises on dimensional grounds. Plugging in numbers, one finds that $\frac{d E}{d x}=10^{12} \mathrm{D} \mathrm{MeVg}{ }^{-1} \mathrm{~cm}^{2}$.

For EDM's of $10^{-16}$ e-cm, this gives an energy loss which is approximately $10^{-4}$ of the usual energy loss for a charged particle. This is challenging for experimenters, but 
not impossible, requiring specialized detectors. For example, if the heavy neutrino is produced in the decay of a charged lepton, then it would be produced in coincidence with a real or virtual $\mathrm{W}$, which would eliminate backgrounds. Note that the event rate, for $D=10^{-16} \mathrm{~cm}$, is huge, corresponding to 100,000 events for an integrated luminosity

of $100 \mathrm{fb}^{-1}$. This is several events per hour, and thus a specialized detector located on the outside of the main detectors could suffice, without needing a full $4 \pi$ coverage (note that the N's will emerge back-to-back). More importantly, however, is that initial and final state radiation will be huge, and thus standard searches for single-photon plus missing energy events will discover a huge signal, which could then be followed by a specialized search for direct detection. We now turn to the possibility of detection through single photon events.

\section{Detection through single photon events}

In the standard model, the process $e^{+} e^{-} \rightarrow \nu \bar{\nu} \gamma$ has been calculated and used to determine the number of light neutrinos [18, 19]. The signature of the process is a single photon from initial state radiation plus missing energy. The process didn't rule out the existence of heavy neutrinos. Similarly, the process $e^{+} e^{-} \rightarrow N \bar{N} \gamma$ can be used to study the effects of large EDM of heavy neutrinos. Now the single photon can also come from final state radiation because of the large EDM.

The interaction of heavy neutrinos with the photon is $-i e \bar{N} D \sigma_{\mu \nu} \gamma_{5} q^{\nu} N A^{\mu}$, where q is the momentum transfer. The EDM is defined to be $e D\left(q^{2}=0\right)$. In this case, the photon momenta at the relevant vertices are different, and are certainly not zero. However, we presume that $D\left(q^{2}\right)$ does not vary too rapidly with $q^{2}$, as is the case in which the momentum transfer is not substantially greater than the masses of the particles in the loop. The feynman diagrams are listed in Fig. 6. The contribution due to the exchange 

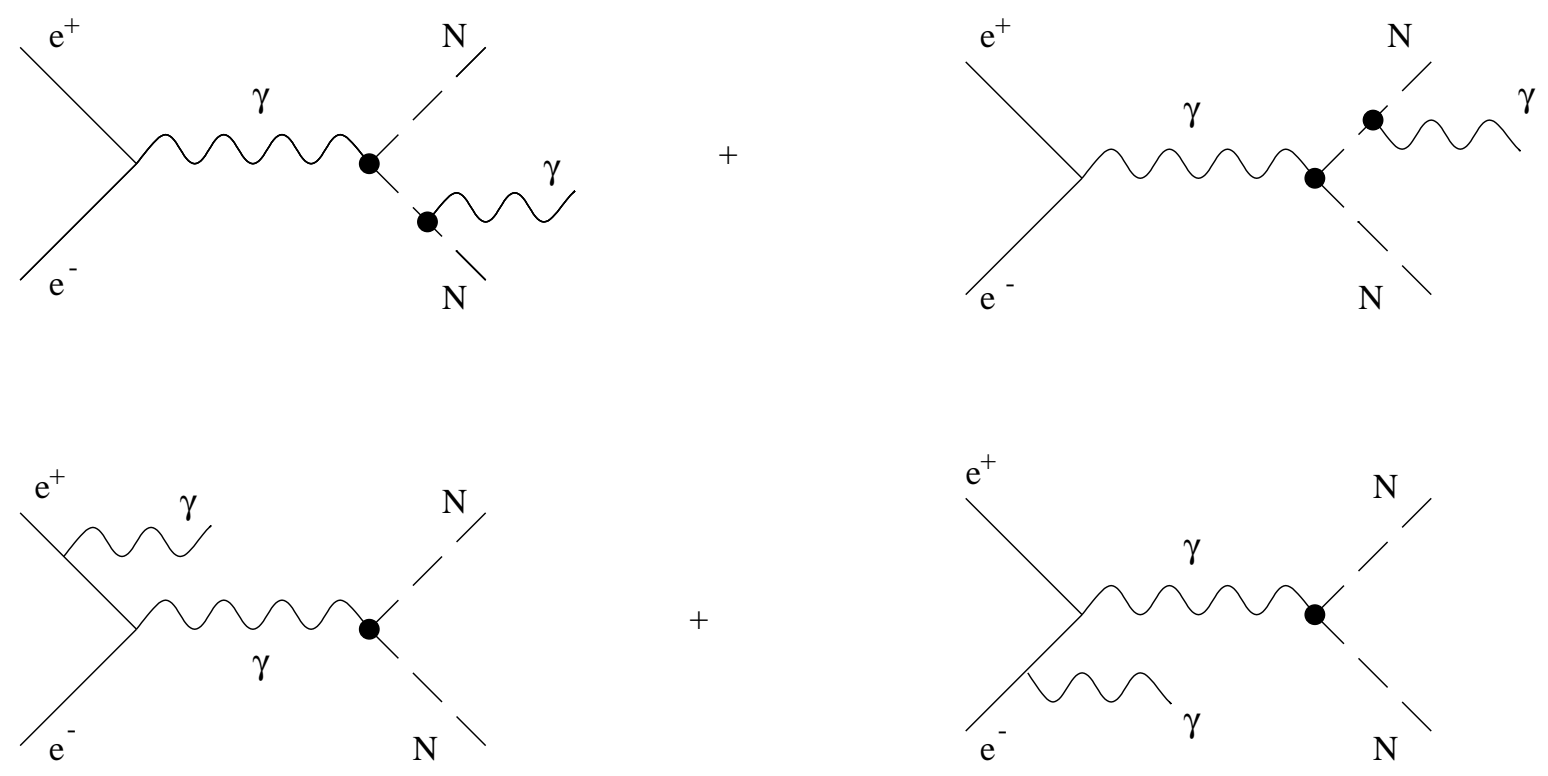

Figure 6: Feynman diagrams which contribute to the process $e^{+} e^{-} \rightarrow N \bar{N} \gamma$.

of the $\mathrm{Z}$ boson is neglected here since it is numerically small 20].

The calculation is straightforward. It is clear that the rate is the sum of terms proportional to $D^{2}, D^{3}$ and $D^{4}$. The contributions proportional to $D^{2}, D^{3}$ and $D^{4}$ are presented separately in Fig. 7 for $D=10^{-16}$ e-cm. For different $D$ 's, these curves scale appropriately. One can see that for $D<6 \times 10^{-16} \mathrm{e}-\mathrm{cm}$, expected in realistic models, the $D^{2}$ term dominates. Because of this scaling, instead of falling as $1 / s$, the cross section reaches a constant (it would grow as $s$ if the $D^{4}$ term dominated. As discussed above, however, it does not reach the level at which unitarity is a big concern. The total cross section is then given, to a good approximation, by the $D^{2}$ term in Fig. 0 .

The cross section will be singular as the $\left|\cos \theta_{\gamma}\right|$ tends to 1 . We have used cuts in the photon energy and in the angle: $\frac{E_{\gamma}}{\sqrt{s}} \geq 0.1$ and $\left|\cos \theta_{\gamma}\right| \leq 0.94$. For example, at $\sqrt{s}=500$ $\mathrm{GeV}$, the cross section is about $1.5 \times 10^{-4} \mathrm{nb}$ (and scales as $D^{2}$ ). This is a very large event rate, corresponding to about an event every few minutes (for $D=10^{-16} \mathrm{e}-\mathrm{cm}$ ) at a linear collider with a luminosity of $3 \times 10^{34} \mathrm{~cm}^{-2} \mathrm{sec}^{-1}$.

The angular distribution is also important. It is shown in Fig. 8 for $D=10^{-16} \mathrm{e}-\mathrm{cm}$, 


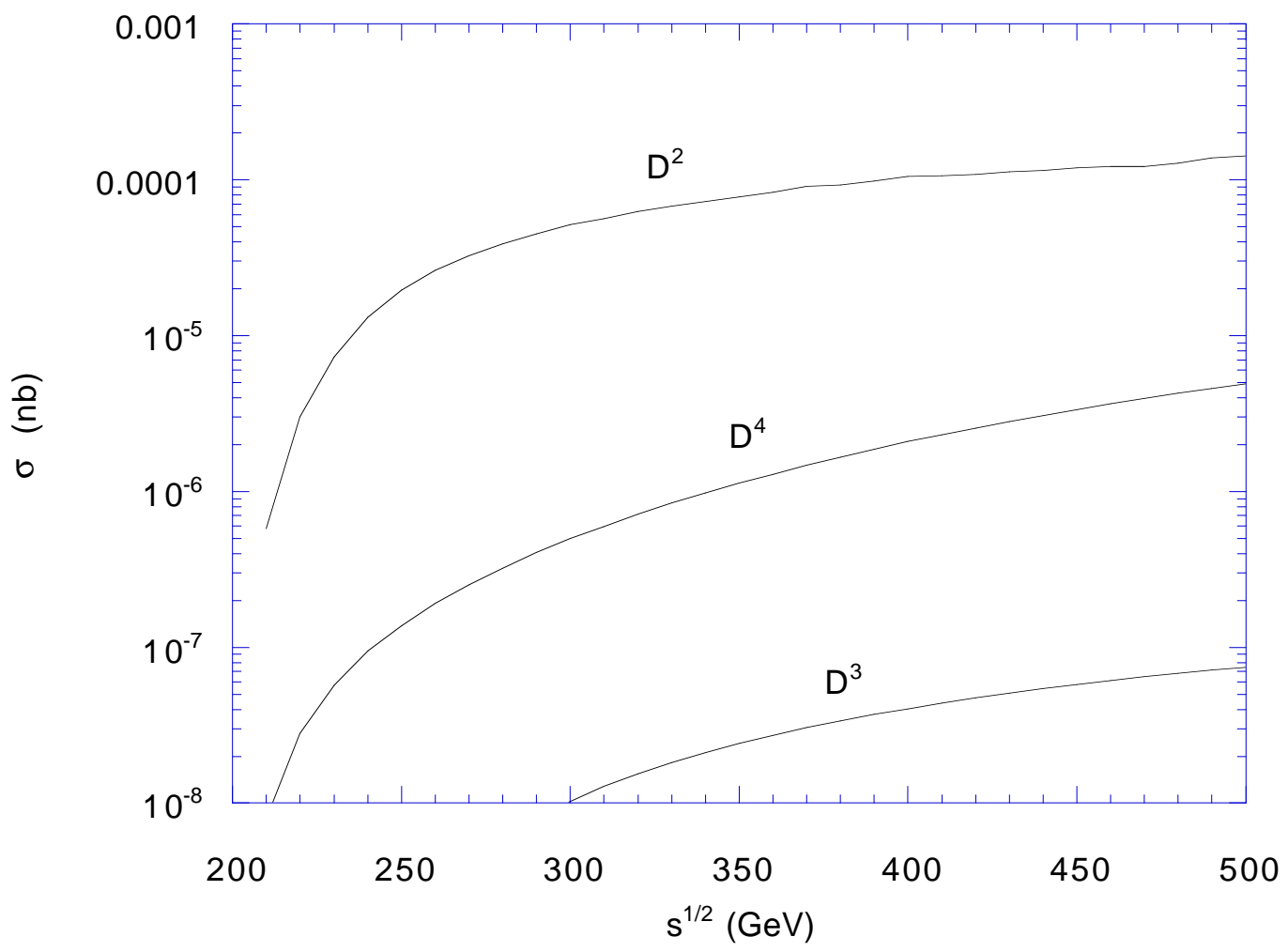

Figure 7: Different contributions to the total cross section for the process $e^{+} e^{-} \rightarrow N \bar{N} \gamma$ with the heavy neutrino mass of $100 \mathrm{GeV}$ and $D=10^{-16} e \mathrm{~cm}$. For a positive EDM, the $D^{3}$ term is negative; the absolute value is shown. 


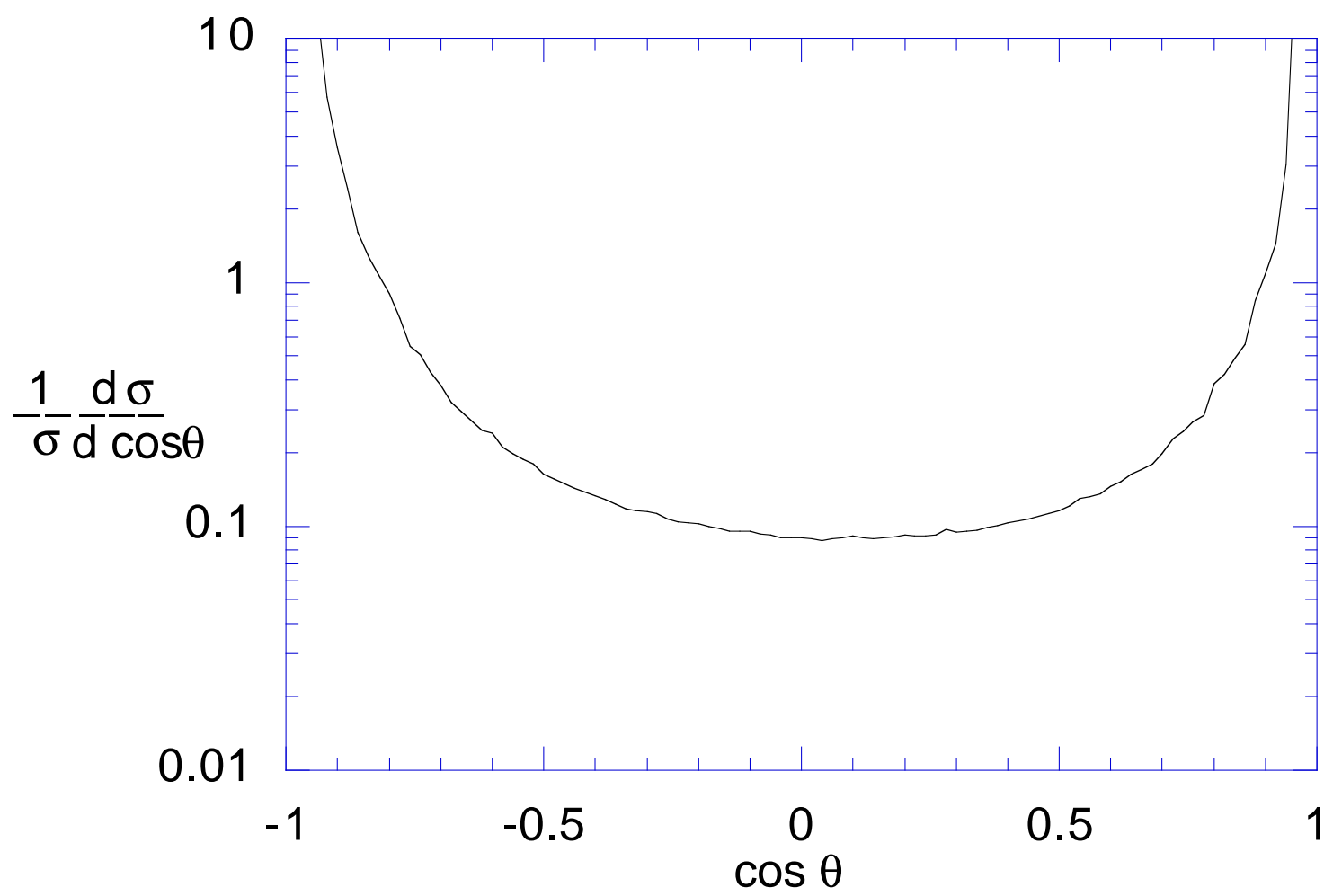

Figure 8: Angular distribution of the cross section with the heavy neutrino mass of 100 $\mathrm{GeV}$ and $D=10^{-16} e \mathrm{~cm}$, and $s^{1 / 2}=500 \mathrm{GeV}$.

and the resulting forward-backward asymmetry is shown as a function of $D$ in Fig. 9. The angular distribution of the Standard Model is nearly forward-backward symmetric, but in this case there is a sizable asymmetry. For much of the parameter space, the asymmetry is -0.48 .

Clearly, the first signature of an EDM for a heavy neutrino will be a very large event rate for single photon events, with the forward-backward asymmetry measurement giving a confirmation of this particular interpretation of such a large rate.

\section{$5 \quad$ Astrophysical and Cosmological Significance}

One of the greatest mysteries in cosmic ray astronomy concerns the existence of cosmic rays with energies above the GZK bound[21]. A substantial number of cosmic rays with 


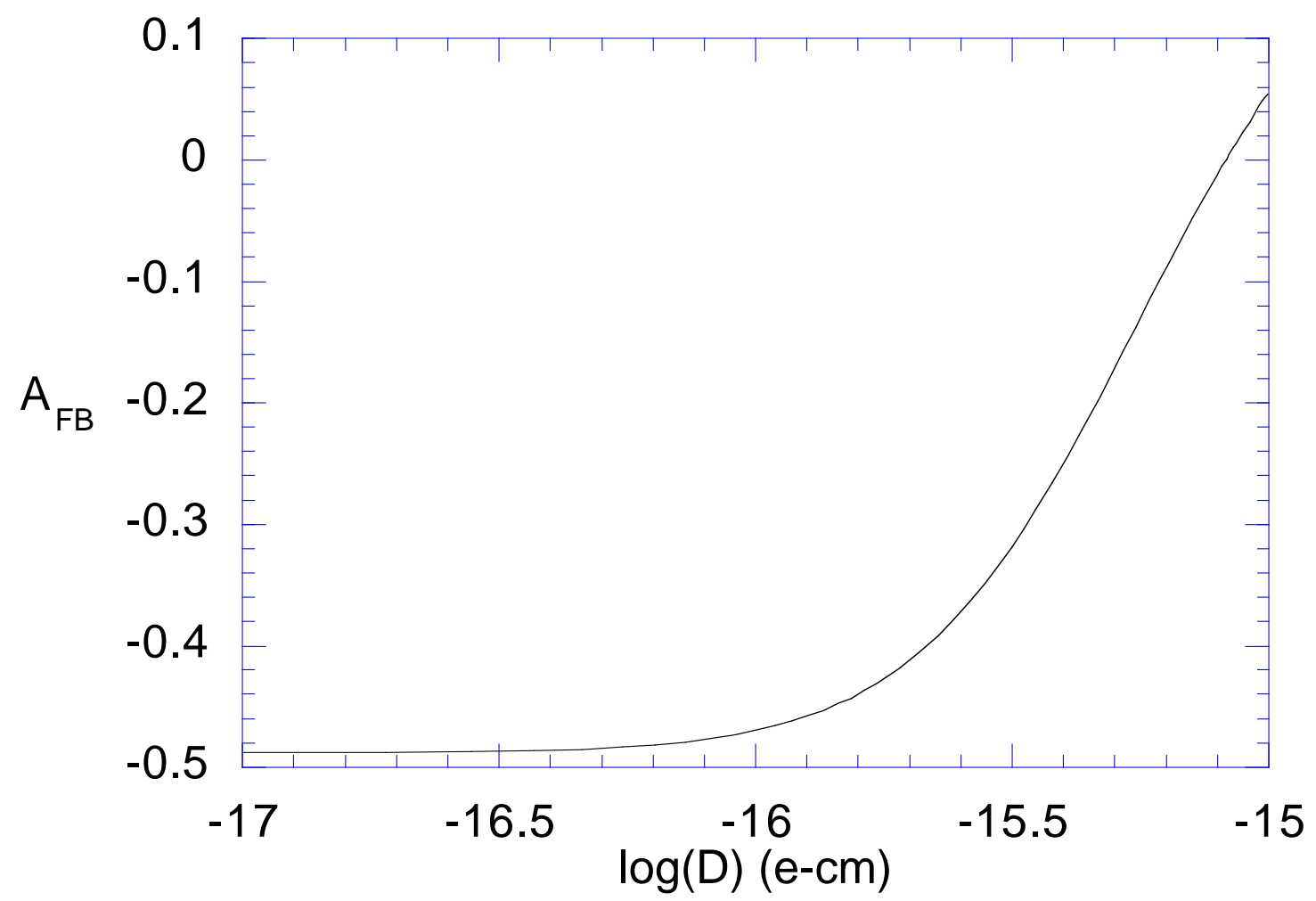

Figure 9: The forward-backward asymmetry as a function of the EDM. 
energies well above $10^{20} \mathrm{eV}$ have been observed, interacting in the upper atmosphere. Yet a charged particle's mean free path through cosmic microwave background, at these energies, is only about 50 megaparsecs, and no sources of such high energy particles are known to exist within this distance. Neutral particles will not have this problem. However, the fact that the cosmic rays shower high up in the atmosphere implies that the cross-section must be of strong interaction strength. The only strongly interacting long-lived neutral particle is the neutron, yet its lifetime is too short. Neutrinos will not interact high in the atmosphere, if they have conventional weak interactions.

Several papers 22 have considered the possibility that neutrino interactions might become strong at very high energies, due to exchange of Kaluza-Klein gravitons 22, 23. One might wonder whether heavy neutrinos with a sufficiently large EDM might interact strongly enough in the upper atmosphere, but not interact strongly with the microwave background. Are these candidates for the ultra-high energy cosmic rays? One can redo the calcuation described above concerning ionization loss in a detector, but now looking at the ultrarelativistic limit. This is a tedious, but straightforward calculation.

In this case, the cross section can not be high enough. Recall that the EDM is defined at $q^{2}=0$. However, here one would expect $q^{2}$ to be enormous. Certainly, in the first few interactions, it will be much larger than the mass-squared of the particles in the loop that produces a large EDM in the first place. As a result, the effective EDM will be much smaller, and the interaction will not be strong. These heavy neutrinos will not interact high in the atmosphere, and may not interact much more than conventional high energy neutrinos.

Are there any other potential astrophysical or cosmological effects? One possibility concerns the generation of the baryon asymmetry. Suppose the heavy neutrino decays radiatively into light neutrinos. Then the huge EDM, which violates $\mathrm{CP}$, may cause the decay rate into neutrinos to be different than the decay rate into antineutrinos. This 
would result in a large lepton asymmetry. Since non-perturbative sphaleron interactions can convert a lepton asymmetry into a baryon asymmetry, this would result in a baryon asymmetry. The reader is referred to the article of Riotto and Trodden[24 for a nice review. This possibility is currently under investigation.

\section{Conclusions}

In this Article, we have explored the possibility that a heavy neutrino could have a huge electric dipole moment. It has been shown that plausible models exist that would lead to a dipole moment as large as $10^{-16} \mathrm{e}-\mathrm{cm}$, and that this would dramatically alter the electromagnetic properties of these neutrinos. The first signature of such a neutrino would come from a large enhancement of the single photon plus missing energy event rate. Then, one could actually look for the ionization track of these neutrinos in specially designed detectors. Cross sections and angular distributions are calculated as a function of the electric dipole moment. While these neutrinos can not explain the ultra high energy cosmic rays, they may have interesting implications for baryogenesis.

\section{Acknowledgments}

We thank Mariana Frank as one of us (Nie) is now doing postdoctoral research under

her supervision. We also are extremely grateful to John Ralston, for repeatedly warning us that the EDM at high energies would be softened, making it unlikely that these neutrinos could be the UHE cosmic rays. We finally believed him. We also thank Jack Kossler and Chris Carone for many useful discussions. This work is supported by the National Science Foundation grant number PHY-9900657. 


\section{References}

[1] Y. Semertzidis et al., hep-ph/0012087 [in Proceedings of the international Workshop on High Intensity Muon Sources (HIMUS99), KEK, Japan, 1999 (to be published)].

[2] J. Bailey et al., J. Phys. G4, 345 (1978).

[3] E. Commins et al., Phys. Rev. A50, 2960 (1994); K. Adullah et al., Phys. Rev. Lett. 65, 2347 (1990).

[4] R. Escribano and E. Masso, Phys. Lett. B395, 369 (1997).

[5] D. E. Groom et al., Particle Data Group, Eur. Phys. J. C15, 1 (2000).

[6] F. Hoogeveen, Nucl. Phys. B341, 322 (1990) ; I. B. Khiplovich and M. Pospelov, Sov. J. Nucl. Phys. 53, 638 (1991).

[7] V. Barger, A. Das and C. Kao, Phys. Rev. D55, 7099 (1997), and references therein.

[8] W. Berbreuther, A. Brandenburg and P. Overmann, Phys. Lett. B391, 413 (1997)

[9] J. Nieves, D. Change and P. Pal, Phys. Rev. D33, 3324 (1986); J. Valle, Phys. Lett. B138, 155 (1984) ; H. Y. Cheng, Phys. Rev. D34, 2794 (1986); K. S. Babu, B. Dutta and R. N. Mohapatra, Phys. Rev. Lett. 85, 5064 (2000).

[10] F. del Aguila, M. B. Gavela, J. A. Grifols and A. Mendez, Phys. Lett. B126, 71 (1983) .

[11] K. S. Babu, S. M. Barr and Ilja Dorsner, Phys. Rev. D64, 053009 (2001).

[12] M. Sher, Phys. Rev. Lett. 87, 161801 (2001) .

[13] W. Bernreuther, T. Schroder and T. N. Pham, Phys. Lett. B48, 78 (1993) . 
[14] A. Czarnecki and W. J. Marciano, Phys. Rev. D64, 01 (20013014).

[15] W. Bernreuther, O. Nachtmann and P. Overmann, Phys. Rev. D48, 78 (1993).

[16] P. H. Frampton, P. Q. Hung and M. Sher, Phys. Rep. 330, 263 (2000) .

[17] J. D. Jackson, Classical Electrodynamics (Wiley, New York, 1975), 2nd ed., Chap. 13.

[18] E. Ma and J. Okada, Phys. Rev. Lett. 41, 287 (1978).

[19] K. J. F. Gaemers, R. Gastmans and F. M. Renard, Phys. Rev. D19, 1605 (1979).

[20] Note that the cross-section for mu-pair production via $\gamma$-exchange is bigger than that via $Z$-exchange, at high energies, by a factor of $256 \sin ^{4} \theta_{W} \cos ^{4} \theta_{W}$, which is approximately ten.

[21] K. Greisen, Phys. Rev. Lett. 16, 748 (1966); G. T. Zatsepin and V. A. Kazmin, Sov. Phys. JETP Lett. 4, 78 (1966); J. Linsley, Phys. Rev. Lett. 10, 146 1963; D. Burd et al., Phys. Rev. Lett. 71, 3401 (1993); Astrophys. J. 424, 491 (1994); M. Takedu et al. , Phys. Rev. Lett. 81, 1163 (1998) and astro-ph/9902239; G. E. Romero, Jorge A. Combi, Luis A. Anchordoqui and S. E. P. Bergliaffa, Astropart. Phys. 5, 279 (1996); V. Berezinsky, M. Kachelriess and A. Vilenkin, Phys. Rev. Lett. 79, 4302 (1997); G. R. Farrar and Tsvi Piran, Phys. Rev. Lett. 84, 3527 (2000);

[22] See John P. Ralston, P. Jain, Douglas W. McKay and S. Panda, Talk given at 7th Conference on Intersections Between Particle and Nuclear Physics (CIPANP 2000), Quebec City, Quebec, Canada, 22-28 May 2000 and P. Jain, Douglas W. McKay, S. Panda and John P. Ralston, Phys. Lett. B484, 267 (2000) for detailed summaries and an extensive list of references. 
[23] N. Arkani-Hamed, S. Dimopoulos and G. Dvali, Phys. Lett. B429, 263 (1998) .

[24] A. Riotto and M. Trodden, Ann. Rev. Nucl. Part. Sci. 49, 35 (1999). 